\title{
Edge Electron Gas
}

\author{
Walter Kohn and Ann E. Mattsson \\ Department of Physics, UCSB, CA 91306-9530
}

\begin{abstract}
The uniform electron gas, the traditional starting point for density-based manybody theories of inhomogeneous systems, is inappropriate near electronic edges. In its place we put forward the appropriate concept of the edge electron gas.
\end{abstract}

Since the work of Thomas and Fermi (TF) [1] through the earliest papers on density functional theory (DFT) [2] up to ongoing work involving density gradients [3], the uniform electron gas has been the starting point of density-based approximate theories of inhomogeneous systems. However, real physical systems have electronic edge regions where effective single particle wave functions evolve from oscillatory to evanescent, and where clearly approximations starting from a uniform electron gas are ill-founded.

The edge surface $S$, for a given system, may be precisely defined by

$$
v_{\text {eff }}(\boldsymbol{r})=\mu,
$$

where $v_{\text {eff }}(\boldsymbol{r})$ is the exact self-consistent potential of the Kohn-Sham (KS) equations [2] and $\mu$ is the chemical potential, half-way between the highest occupied and the lowest unoccupied KS orbital energies (In what follows we take $\mu=0)$. Outside of $S$ all occupied KS orbitals decay exponentially (See Fig. (1).

Much interesting science involves precisely such edge regions (See Fig. 1), such as ionization energies of the outermost electrons, molecular binding, surface energies etc. For these regions, in place of the uniform electron gas, we discuss in this paper the new concept of the edge electron gas, which is valid in the edge region near $S$ [

The edge gas concept is based on the principle of "nearsightedness" put forward in [5]: The local electronic structure near a point $\boldsymbol{r}$, while strictly speaking requiring a knowledge of the density $n\left(\boldsymbol{r}^{\prime}\right)$, or equivalently of the effective potential $v_{\text {eff }}\left(\boldsymbol{r}^{\prime}\right)$, everywhere, in fact is largely determined by $v_{\text {eff }}\left(\boldsymbol{r}^{\prime}\right)$ for $\boldsymbol{r}^{\prime}$ near $\boldsymbol{r}$. Nearsightedness applies just as much to the edge region as to the interior region far inside $S$.

This principle has been applied to the density $n(\boldsymbol{r})$ and to the one particle density matrix [5]. In the present paper we also apply it to the exchange energy $E_{x}$ as follows: We write

$$
E_{x}=-\frac{e^{2}}{2} \int n(\boldsymbol{r}) R_{x}^{-1}(\boldsymbol{r}) d \boldsymbol{r} .
$$

where $R_{x}^{-1}(\boldsymbol{r})$, the inverse radius of the exchange hole, is defined by

$$
R_{x}^{-1}(\boldsymbol{r}) \equiv-\int \frac{n_{x}\left(\boldsymbol{r} ; \boldsymbol{r}^{\prime}\right)}{\left|\boldsymbol{r}-\boldsymbol{r}^{\prime}\right|} d \boldsymbol{r}^{\prime}
$$

and $n_{x}\left(\boldsymbol{r} ; \boldsymbol{r}^{\prime}\right)$ is the exchange hole density which satisfies $\int n_{x}\left(\boldsymbol{r} ; \boldsymbol{r}^{\prime}\right) d \boldsymbol{r}^{\prime}=-1$.

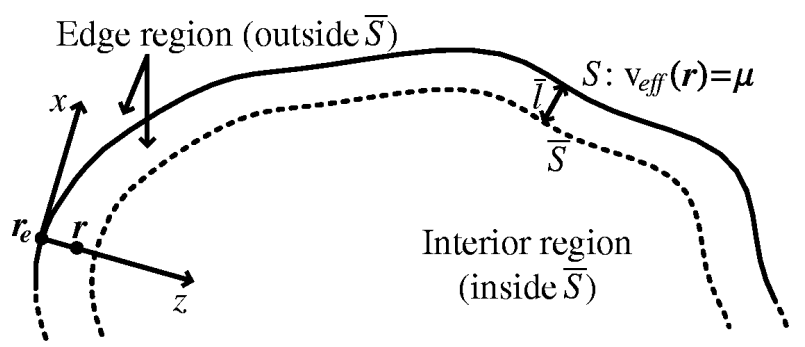

FIG. 1. Interior and Edge regions of a bounded system. $S$ is the nominal dividing surface (-). $\left.\bar{S}(--)^{-}\right)$is the physical dividing surface with $\bar{l}=\gamma l . l$ is defind in Eq. (6) and $1<\gamma<3$.

For a consideration of the electronic structure at a point $\boldsymbol{r}$ near $S$ we first drop a perpendicular to the nearest point $\boldsymbol{r}_{e}$ on $S$, take $\boldsymbol{r}_{e}$ as origin of coordinates and the $z$-axis through $\boldsymbol{r}$ (See Fig. 1.); thus $\boldsymbol{r}=(0,0, z)$. Near $\boldsymbol{r}_{e}$ we have, up to first order in $(x, y, z)$,

$$
v_{e f f}(\boldsymbol{r})=-F z . \quad \text { (Airy Gas) }
$$

This leads to the concept of the Airy gas (AG), the simplest version of the edge gas (Fig. 27a). The AG is a principal subject of this paper. Of course only the properties of the AG near the edge at $z=0$ are of physical interest.

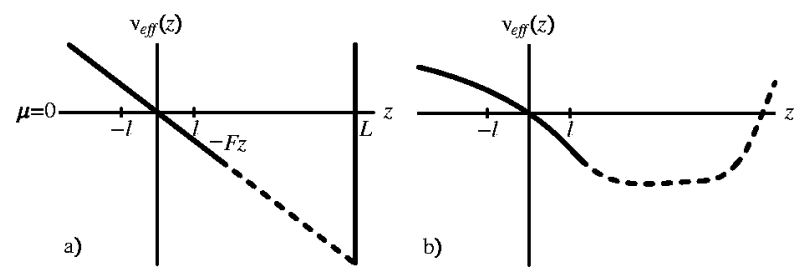

FIG. 2. a) The Airy gas: a strictly linear $v_{\text {eff }}$, with a hard wall at the distant point $L$. b) The edge gas: a typical $v_{\text {eff }}(z)$ near the edge at $z=0$. All levels up to $\mu=0$ are occupied.

In many cases $v_{\text {eff }}$ varies rapidly along $z$ but slowly along $x$ and $y$, i. e. $\kappa l \lesssim 1$, where $l$ is the nominal surface "thickness", defined in Eq. (6) below, and $\kappa$ is the curvature of $S$ at $\boldsymbol{r}_{e}$. This leads to the more general concept of the Edge Gas (EG), 


$$
v_{e f f}(\boldsymbol{r})=v_{e f f}(z) ; \quad v_{\text {eff }}^{\prime}(0)=-F, \quad(\text { Edge Gas })
$$

(Fig. 2b). The EG can be refined by including curvature corrections [6].

There have been extensive previous studies of several model systems, notably by V. Sahni, A. G. Eguiliz and their collaborators [7], of electrons moving in effective potentials $v_{\text {eff }}(z)$ with an electronic edge, say at $z=0$. Their common features are: $v_{\text {eff }}(-\infty)>\mu$ and $v_{\text {eff }}(+\infty)=$ constant $<\mu$. The differences between earlier work and the present contribution are: (i) Our emphasis on the locality of the electronic edge structure, i. e. its independence of the behavior of $v_{e f f}(\boldsymbol{r})$ far from the edge; (ii) our detailed analysis of the simplest universal edge model, $v_{\text {eff }}(z)=-z \quad(-\infty<z<+\infty)$ which, by appropriate scaling, provides a first approximation for dealing with general $3 D$ systems with edges; (iii) preliminary discussions of further improvments.

The Edge Electron Gas: We now consider the situation in Fig. 2 b where the effective potential is independet of $x$ and $y$ but generic in $z$.

The surface "thickness" $l$ and the corresponding energy $\epsilon$ are defined uniquely, up to constant factors, by

$$
l \equiv\left(\frac{\hbar^{2}}{2 m F}\right)^{1 / 3} ; \quad \epsilon \equiv\left(\frac{\hbar^{2} F^{2}}{2 m}\right)^{1 / 3},
$$

where $F \equiv\left|v_{\text {eff }}^{\prime}(0)\right|$.

The normalized eigenfunctions of the KS equations [2] are

$$
\psi_{\nu}(x, y, z)=\varphi_{j}(z) \frac{1}{A^{1 / 2}} e^{i\left(k_{1} x+k_{2} y\right)} ;\left(\varphi_{j}, \varphi_{j^{\prime}}\right)=\delta_{j j^{\prime}}
$$

where $\nu \equiv\left(j, k_{1}, k_{2}\right) ; k_{i} L_{i}=2 \pi m_{i}(i=1,2)$ and $A \equiv$ $L_{1} L_{2}$ is the cross-sectional area. The functions $\varphi_{j}(z)$ satisfy

$$
\left(-\frac{\hbar^{2}}{2 m} \frac{d^{2}}{d z^{2}}+v_{e f f}(z)-\epsilon_{j}\right) \varphi_{j}(z)=0
$$

and the boundary conditions

$$
\varphi_{j}(-\infty)=\varphi_{j}(+\infty)=0
$$

The $\epsilon_{j}$ are negative and, for each $\epsilon_{j},\left(k_{1}, k_{2}\right)$ satisfy the inequality

$$
\epsilon_{\nu} \equiv \frac{\hbar^{2}}{2 m}\left(k_{1}^{2}+k_{2}^{2}\right)+\epsilon_{j} \leq 0 .
$$

The density of the EG is given by

$$
n(z)=2 \sum_{j} \varphi_{j}^{2}(z) w_{j} ; \quad w_{j}=\frac{m}{2 \pi \hbar^{2}}\left|\epsilon_{j}\right|
$$

where the factor 2 accounts for the spin and where $w_{j}$ is the weight factor accounting for the number of occupied wave-vectors $\left(k_{1}, k_{2}\right)$.
The exchange hole density, $n_{x}\left(\boldsymbol{r} ; \boldsymbol{r}^{\prime}\right)$, is defined by

$$
\begin{aligned}
n_{x}\left(\boldsymbol{r} ; \boldsymbol{r}^{\prime}\right) & \equiv-\frac{1}{2} \frac{\left|\rho_{1}\left(\boldsymbol{r} ; \boldsymbol{r}^{\prime}\right)\right|^{2}}{n(\boldsymbol{r})} ; \\
\rho_{1}\left(\boldsymbol{r} ; \boldsymbol{r}^{\prime}\right) & \equiv 2 \sum_{\epsilon_{\nu} \leq 0} \psi_{\nu}(\boldsymbol{r}) \psi_{\nu}^{*}\left(\boldsymbol{r}^{\prime}\right) .
\end{aligned}
$$

Inserting Eq. (7) in Eq. (13) and integrating over $k_{1}$ and $k_{2}$ gives

$$
\rho_{1}\left(\boldsymbol{r} ; \boldsymbol{r}^{\prime}\right)=\frac{1}{\pi} \sum_{j} \varphi_{j}(z) \varphi_{j}\left(z^{\prime}\right) \frac{k_{j} J_{1}\left(k_{j} \rho^{\prime}\right)}{\rho^{\prime}}
$$

where $J_{1}(x)$ is the first order Bessel function and where, without loss of generality, we have set $\boldsymbol{r}=(0,0, z)$, and have expressed $\boldsymbol{r}^{\prime}$ in cylindrical coordinates $\rho^{\prime}, \alpha^{\prime}$ and $z^{\prime}$. $k_{j}$ is the maximum transverse wave number associated with $\varphi_{j}(z)$ given by $k_{j}=\left(2 m\left|\epsilon_{j}\right| / \hbar^{2}\right)^{1 / 2}$. This leads to

$$
\begin{array}{r}
R_{x}^{-1}(\boldsymbol{r})=\frac{1}{\pi n(z)} \int d z^{\prime} \sum_{j} \sum_{j^{\prime}} \varphi_{j}(z) \varphi_{j}\left(z^{\prime}\right) \varphi_{j^{\prime}}(z) \varphi_{j^{\prime}}\left(z^{\prime}\right) \times \\
\times(\Delta z)^{-3} g\left(k_{j} \Delta z, k_{j^{\prime}} \Delta z\right),
\end{array}
$$

where $\Delta z=\left|z-z^{\prime}\right|$ and the universal function $g\left(s, s^{\prime}\right)$, independent of $v_{e f f}(z)$, is given by

$$
g\left(s, s^{\prime}\right) \equiv s s^{\prime} \int_{0}^{\infty} d t \frac{J_{1}(s t) J_{1}\left(s^{\prime} t\right)}{t \sqrt{1+t^{2}}} .
$$

We have calculated $g\left(s, s^{\prime}\right)$ numerically and present a contour plot in Fig. 3. Further details about $g\left(s, s^{\prime}\right)$ are available from A. E. Mattsson.

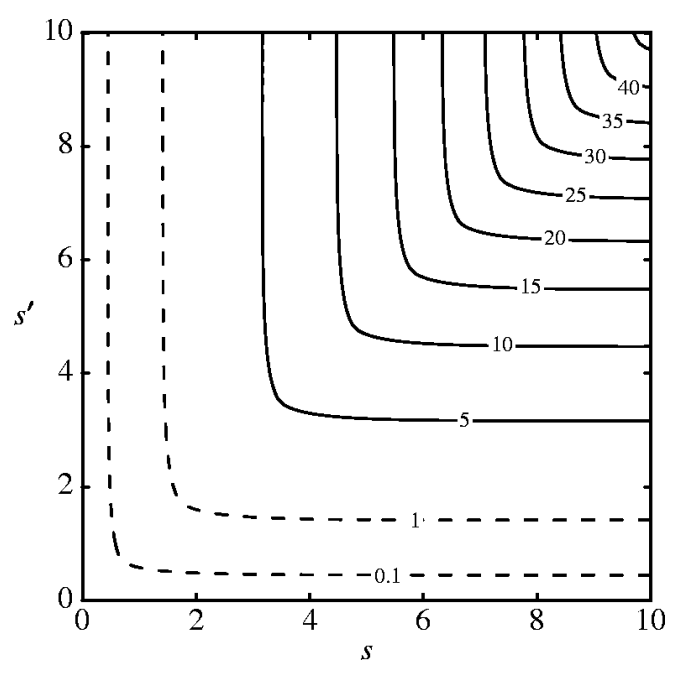

FIG. 3. The universal function $g\left(s, s^{\prime}\right)$ of Eq. (16).

The Airy Gas: We now consider the simplest model of an edge electron gas, the Airy Gas (AG) of Fig. 2a, in which the potential has the strictly linear form 


$$
v_{e f f}(z)=\left\{\begin{array}{ccc}
-F z & -\infty<z<L & (F>0) \\
+\infty & z \geq L & (L / l \rightarrow \infty)
\end{array}\right.
$$

The normalized eigenfunctions $\varphi_{j}(z)$ satisfy

$$
\begin{aligned}
& \left(-\frac{\hbar^{2}}{2 m} \frac{d^{2}}{d z^{2}}-F z-\epsilon_{j}\right) \varphi_{j}(z)=0, \\
& \varphi_{j}(-\infty)=\varphi_{j}(L)=0 .
\end{aligned}
$$

In suitable variables all the $\varphi_{j}(z)$ are proportional to the Airy function $A i(-z) ; \operatorname{Ai}(\mathrm{z})$ satisfies

$$
\left(-\frac{d^{2}}{d z^{2}}+z\right) A i(z)=0, \quad A i(+\infty)=0
$$

The function $\Phi(z) \equiv A i(-z)$ is shown in Fig. 1 .

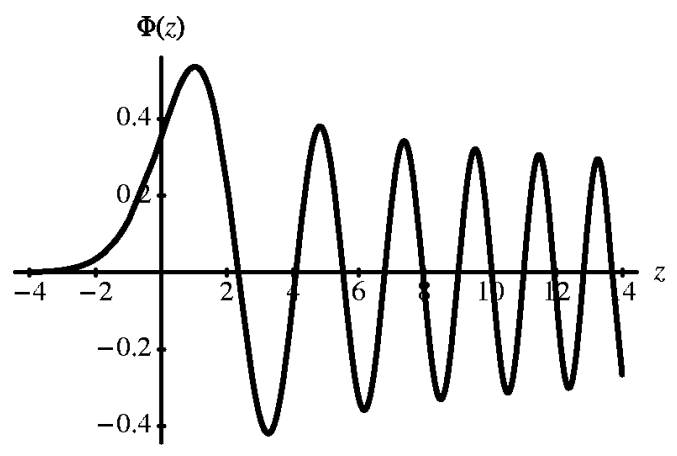

FIG. 4. The function $\Phi(z) \equiv A i(-z)$ [8].

Relevant properties of $\Phi(z)$ are [8]:

$$
\left\{\begin{array}{l}
\Phi(0)=0.35503 ; \quad \Phi^{\prime}(0)=0.25882 \\
z \rightarrow-\infty: \Phi(z) \approx \frac{1}{2 \pi^{1 / 2}|z|^{1 / 4}} e^{-\frac{2}{3}|z|^{3 / 2}} \\
z \rightarrow+\infty: \Phi(z) \approx \frac{1}{\pi^{1 / 2} z^{1 / 4}} \sin \left(\frac{2}{3}|z|^{3 / 2}+\frac{\pi}{4}\right)
\end{array}\right.
$$

In the $\mathrm{AG}$ all $3 D$ states with energy up to $\mu=0$ are occupied (Fig. 2a). The AG is completely characterized by the length $l$ and the energy $\epsilon$ in Eq. (6). In units of $l$ and $\epsilon$ all AG's are identical. We shall later verify that for $z>>l$ the AG behaves like a locally homogeneous gas whose density is $n(z)=\left(3 \pi^{2}\right)^{-1}\left(2 m F / \hbar^{2}\right)^{3 / 2} z^{3 / 2}$. Thus the "thickness" of the edge region is $\sim l$. The $\varphi_{j}(z)$ contributing to the edge electron density have $\epsilon_{j}$ of the order $-\epsilon$, much less, in absolute value, than the lowest eigenvalue $(\sim-F L)$. These small $\epsilon_{j}$, obtainable from the boundary condition $\varphi_{j}(L)=0$, and the $\varphi_{j}(z)$ are given by

$$
\epsilon_{j}=-j\left(\frac{l}{L}\right)^{1 / 2} \pi \epsilon ; \quad \varphi_{j}(z)=\frac{\pi^{1 / 2}}{(L l)^{-1 / 4}} \Phi\left(\frac{z}{l}+\frac{\epsilon_{j}}{\epsilon}\right) .
$$

We note that the $\epsilon_{j}$ are equally spaced and that successive $\varphi_{j}(z)$ 's are identical except for a small shift, $l(l / L)^{1 / 2} \pi$, to the right.

Using Eqs. (22) in Eq. (11) we obtain for the density

$$
\begin{aligned}
n(z) & =l^{-3} n_{0}(\zeta), \quad(\zeta \equiv z / l) \\
n_{0}(\zeta) & \equiv \frac{1}{2 \pi} \int_{0}^{\infty} \Phi^{2}\left(\zeta-\zeta^{\prime}\right) \zeta^{\prime} d \zeta^{\prime}
\end{aligned}
$$

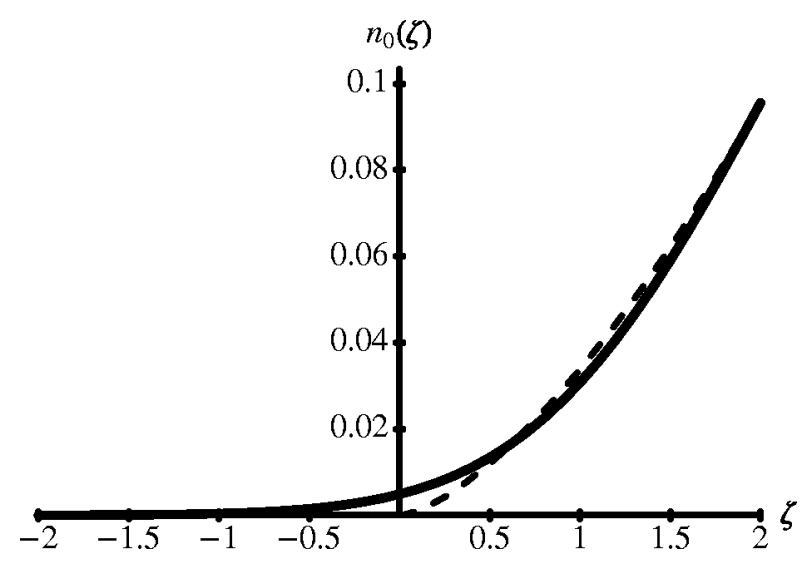

FIG. 5. The density of the Airy gas, exact (-) and in the Thomas Fermi approximation (- - ).

Fig. 2 shows the exponential decay for $\zeta<<-1$. For $\zeta>>1$

$$
n_{0}(\zeta)=\frac{1}{3 \pi^{2}} \zeta^{3 / 2}-\frac{1}{16 \pi^{2}} \zeta^{-3 / 2} \sin \left(\frac{4}{3} \zeta^{3 / 2}\right)+\cdots .
$$

The first term is identical with the Thomas Fermi result (See Fig. 5); the second describes Friedel oscillations, due to the edge at $z=0$.

Exchange: Inserting the appropriate expressions for the AG, Eqs. (22), into Eq. (15) gives

$$
R_{x}^{-1}(z)=l^{-1} R_{x 0}^{-1}(\zeta)
$$

where

$$
\begin{aligned}
& R_{x 0}^{-1}(\zeta)=\frac{1}{\pi n_{0}(\zeta)} \times \\
& \int_{-\infty}^{\infty} d \zeta^{\prime} \int_{0}^{\infty} d \epsilon \int_{0}^{\infty} d \epsilon^{\prime} \Phi(\zeta-\epsilon) \Phi\left(\zeta^{\prime}-\epsilon\right) \Phi\left(\zeta-\epsilon^{\prime}\right) \Phi\left(\zeta^{\prime}-\epsilon^{\prime}\right) \times \\
& \quad \times\left|\zeta-\zeta^{\prime}\right|^{-3} g\left(\sqrt{\epsilon}\left|\zeta-\zeta^{\prime}\right|, \sqrt{\epsilon^{\prime}}\left|\zeta-\zeta^{\prime}\right|\right)
\end{aligned}
$$

In Fig. 6 we see the exact $R_{x 0}^{-1}(\zeta)$ for the AG compared with its local density approximation (LDA) result

$$
\left(R_{x 0}^{-1}(\zeta)\right)_{L D A}=3 / 2(3 / \pi)^{1 / 3}\left(n_{0}(\zeta)\right)^{1 / 3} .
$$




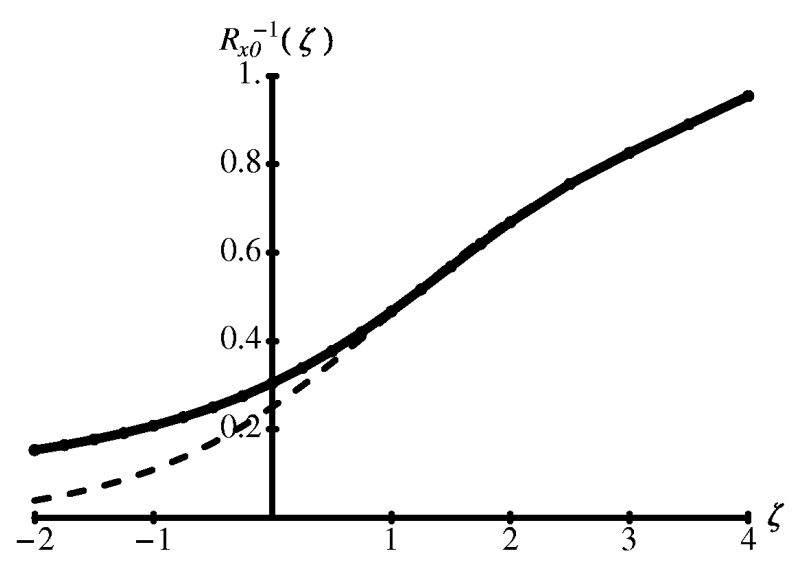

FIG. 6. The inverse radius of the exchange hole of the Airy Gas: (-) exact, (- - ) LDA.

As expected the LDA exchange is much too small for $z / l<-1$, but accurate for $z / l \geq 2$. For $\zeta \rightarrow-\infty$, $R_{x 0}^{-1}(\zeta) \rightarrow \frac{1}{2} \zeta^{-1}$

For the AG the exchange hole density has been calculated numerically and is shown for $\boldsymbol{r}=(0,0,0)$ in Fig. (7).

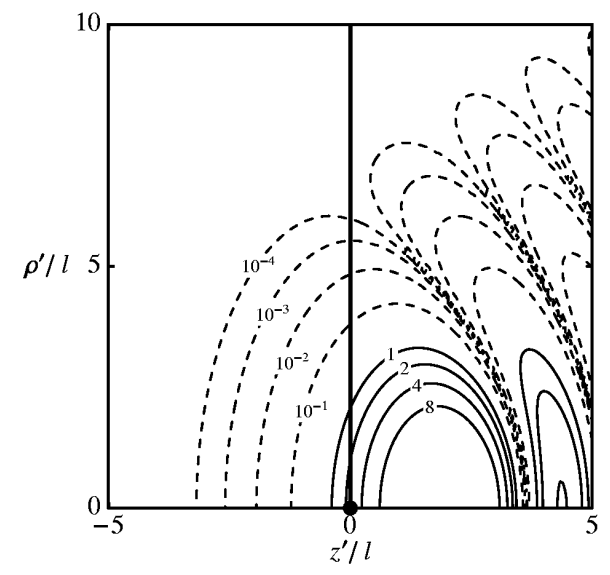

FIG. 7. The exchange hole density $n_{x}\left(\boldsymbol{r}^{\prime}\right)$ of the Airy Gas for $\boldsymbol{r}=(0,0,0)$ (see the black dot). The vertical line indicates the edge at $z=0$ where $v_{\text {eff }}(z)=\mu$. The values on the contours are given in units of $10^{-3} l^{-3}$.

This may be compared with the isotropic exchange hole density of a uniform electron gas of density $n$, centered at the point $\boldsymbol{r}$ and given by

$$
n_{x}\left(\boldsymbol{r} ; \boldsymbol{r}^{\prime}\right)=-9 n(\sin \tau-\tau \cos \tau)^{2} /\left(2 \tau^{6}\right),
$$

where $\tau=\left(3 \pi^{2} n\right)^{1 / 3}\left|\boldsymbol{r}-\boldsymbol{r}^{\prime}\right|$.

Application to the Edge Problem of Thomas Fermi Theory: For simplicity we first consider electrons with chemical potential $\mu$ in a spherical effective potential $v_{e f f}(r)$. The edge surface $S$ is a sphere of radius $r_{e}$ given by $v_{\text {eff }}\left(r_{e}\right)=\mu$. The "edge problem" in TF theory is that-incorrectly $-n_{T F}(r) \equiv 0$ for $r>r_{e}$ (Compare Fig. 5).
Following present ideas we consider points $\boldsymbol{r}=(0,0, r)$, where $r \sim r_{e}$, and their reference point on $S, \boldsymbol{r}_{e}=$ $\left(0,0, r_{e}\right)$. Near $r_{e}$ we approximate

$$
v_{e f f}(r)=\mu-F\left(r_{e}-r\right), \quad F \equiv\left|v_{e f f}^{\prime}\left(r_{e}\right)\right|
$$

which, by spherical symmetry, gives

$$
n(r)=l^{-3} n_{0}\left(\left(r_{e}-r\right) / l\right) \quad \text { (edge region) }
$$

in the edge region which we take to be $r>r_{e}-\gamma l$ with $l$ defined in Eq. (6) and $\gamma=2$ (see Fig. 5). In the interior region, $r<r_{e}-\gamma l$, we use the standard $\mathrm{TF}$ result

$$
n(r)=\frac{1}{3 \pi^{2}}\left(\frac{2 m}{\hbar^{2}}\left(\mu-v_{e f f}(r)\right)\right)^{3 / 2} .(\text { interior region) }
$$

At $r=r_{e}-\gamma l$ the two forms approximately agree (See Fig. 5).

For an anisotropic $v_{\text {eff }}(\boldsymbol{r}), F\left(\boldsymbol{r}_{e}\right)\left(\equiv\left|\nabla v(\boldsymbol{r})_{\boldsymbol{r}=\boldsymbol{r}_{e}}\right|\right)$ and thereby $l$ will vary over the surface $S$.

Concluding remarks: The concept of "nearsightness" allows separate consideration of the interior and the edge regions. For the latter this paper begins to develop the concept of the edge gas, so far without correlation effects. The joining of interior and edge regions, using the inverse of the exchange-correlation hole radius, $R_{x c}^{-1}(\boldsymbol{r})$ (cp. Eq. 3), will be described in a forthcoming work.

Computational support by Dr. F. Pikus and financial support by NSF grant DMR93-0801 and STINT are gratefully acknowledged.

[1] L. H. Thomas, Proc. Camb. Phil. Soc. 23, 542 (1927); E. Fermi, Rend. Acad. Naz. Lincei 6, 602 (1927).

[2] P. Hohenberg and W. Kohn, Phys. Rev. 136, B864 (1964); W. Kohn and L. J. Sham, Phys. Rev. 140, A1133 (1965).

[3] J. P. Perdew, K. Burke, and M. Ernzerhof, Phys. Rev. Letters 77, 3865 (1996); A. D. Becke, J. Chem. Phys. 107, 8554 (1997).

[4] A short, preliminary note has appeared in a conference proceedings: W. Kohn in "Electronic Density Functional Theory: recent progress and new directions" edited by J. F. Dobson, G. Vignale, and M. P. Das, Plenum Press (New York) 1998.

[5] W. Kohn, Phys. Rev. Letters 76, 3168 (1996).

[6] W. Kohn and F. Pikus, unpublished.

[7] A. Solomatin and V. Sahni, Annals of Phys. 25997 (1997); A. G. Eguiluz, M. Heinrichsmeier, A. Fleszar, and Werner Hanke, Phys. Rev. Letters 68, 1359 (1992), and references cited therein. (These papers also contain discussions of exchange and correlation effects.)

[8] M. Abramowitz and I. A. Stegun, Handbook of Mathematical Functions (Dover, New York, 1964). 